\title{
Method for Constructing Three-Dimensional Display Model on Battlefield Situation Awareness
}

\author{
Rong Chen, Xian Jin Wang, and Jie Chen
}

\begin{abstract}
Using three-dimensional graphic technology to construct virtual battlefield, realize battlefield situation information visualization, enhance situation awareness fidelity and immersion, and improve battlefield situation awareness naturalness, is an important orientation of C4ISR (Command, Control, Communication, Computer, Intelligence, Surveillance and Reconnaissance) system. For normal three-dimensional model couldn't describe the dynamic changing of virtual scene, we put forward a method constructing and drawing flow of three-dimensional model based on the data model of application domain for large-scale virtual scenes. We validate the feasibility of the method combined with the radar power bubble on electronic jam model.
\end{abstract}

Index Terms-Large-scale virtual scenes, radar power bubble, three-dimensional display model.

\section{INTRODUCTION}

Battlefield information fusion and high-summary is the basis for commander and staff planning operation, making decision, and conducting action. Battlefield situation comprising geography, meteorological, electromagnetic environment, military power deployment, current action, plan, and menace, etc. Quickly presenting battlefield situation to commander in manner of visualize, is very important to take the advantage of battlefield information. Using virtual human-computer interaction means to realize the battlefield situation visualization and enhance situation fidelity and immersion, is an important trend in future command and control system of human-computer interaction [1].

Three-dimensional battlefield situation display software mainly uses 3D (Three-Dimensional) engine or platform convention geometrical model format to display battlefield situation elements. Geometrical model generally uses the following two methods to show the different spatial attribute of the same situation element: 1) using entire model scaling, pose modifying, position offset to realize the spatial description of entire situation element; 2) using partial branch model based on Degree Of Freedom (DOF in short) control, scale, modify pose to reflect the spatial relation of model

Manuscript received in November 10, 2012; revised February 23, 2013.

Chen Rong is with the $13^{\text {th }}$ department of $28^{\text {th }}$ Research Institute of China Electroncs Technology Group Corporation, NanJing, China (e_mail: chenr2001_125@yahoo.com.cn).

Wang Xian Jin is with the Science and Technology on Information Systems Engineering Laboratory, NanJing, China (e-mail: WxjMU@ 163.com).

ChenJie is with the Science and Technology on Information Systems Engineering Laboratory, NanJing, China (e-mail: chenjie600006@126.com). constitution element related to entire model.

In three-dimensional battlefield environment, electromagnetic situation element involves the visual description of radar spatial power sphere (power bubble in short), shrinkage and gap display of power bubble on different disturbance mode and different disturbance intensity [1]. Radar spatial power bubble can be previously constructed by using 3DMax, Multigen Creator, etc. However, radar power sphere boundary changes greatly, dynamically and abnormally on different disturbance mode and intensity. It's difficult to display the radar power sphere boundary through scaling axis and setting DOF control branch relative offset. Fixed boundary spatial model can't meet the display need of model boundary changing dynamically.

3D engine such as OpenGL and Direct3D provides basic point, line, triangle primitive figure drawing interface and the method to construct and draw complex shape figure based on vertex set, line and triangle primitive figure vertex index set[2],[3].

The paper includes two parts. Firstly, the construction and drawing method of universal three-dimensional geometrical model format file are introduced. The reason why universal three-dimensional geometrical model format can't describe dynamically changing radar power sphere spatial boundary is given. The construction and drawing flow of three -dimensional model based on the data model of application domain are put forward. Then, we realize the method on the computer combined with three-dimensional radar power bubble changing model on a kind of electronic jam model. Finally, we validate the feasibility of the idea combined with the conception demo effect pictures.

\section{THREE-DimENSIONAL DisPlay MODEL CONSTRUCTION AND DRAWING BASED ON APPLICATION DOMAIN DATA} MODEL DRIVEN

\section{A. Preliminaries}

Virtual battlefield environment involves construction and displaying large-scale of land, sea, sky, space and electricity military situation element symbols. It is difficult to express dynamically changing situation elements in universal geometrical model format using model building tools. Based on the complex graphics construction ability supported by basic $3 \mathrm{D}$ engine, this paper analyzes the situation element shape structure knowledge of application domain data model and puts forward a general requirement of three-dimensional display model construction based on application domain data model driven. Then, through designing situation element 
extended figures faced to application domain, we realize the three-dimensional expression of moving object track, radar detection beam, radar power sphere on disturbance.

In the following text, we first analyze the limitation of universal model format in expressing situation element and the expression ability of 3D engine. Then, three-dimensional display model design method based on application domain driven is introduced.

\section{B. Analysis of the Constitution and Drawing of three- Dimensional Geometrical Model}

At the present time, there are many kinds of three-dimensional geometrical model formats. The regular commercial formats are: a). $x$ format for Direct3D storing mesh data[4]; b).3ds format for 3DMax describing 3D editor chunk and Key framer chunk[5]; c).obj format of Alias Wavefront company; d).mdl format applied to the Satellite Tool Kit[6]; e).flt format used by Multigen Creator[7] and so on. The regular commercial format model file expresses the three-dimensional geometrical model as a tree structure. The model parts can move based on the DOF constraint by interchanging matrix between frames and components. Actually, the model parts' vertices coordinates and vertices set index that determinate triangles construction do not changed. So commercial model file format can't describe three -dimensional geometrical model based on dynamic changing boundary.

The 3D basic graphics library, which three-dimensional battlefield environment display depends on, is OpenGL or Direct3D generally. OpenGL is a 3D graphics standard API established by SGI which is independent of hardware system. It can directly call $3 \mathrm{D}$ process function oriented to hardware and support network running. OpenGL is an open three-dimensional graphics software package. It's independent of window system and operating system. Application program which based on OpenGL can transplant between various platforms [2]. Direct3D is a sub system of DirectX development library of Microsoft multimedia application software package. It includes a group of the low layer and the high layer API and runs in Windows platform [3]. Microsoft already uses DirectX as a basic component of Windows at the beginning of Vista operating system. This paper mainly introduces the construction and drawing technology of three-dimensional display model based on Direct3D platform.

Two 3D development graphics library(OpenGL and Direct3D) both provide primitive figure drawing interface of basic point, line, triangle set[2],[3]. The model saved in three-dimensional geometrical model file will finally be drawn through basic points, lines, triangles.

\section{Construction and Display Requirement of Application Domain Data Model}

Battlefield situation element displayed in threedimensional battlefield environment associates with requirement and application data item. In Fig 1, based on "application domain data model", three-dimensional scene management object constructs "situation element 3D display model". 3D display model uses "3D graphics drawing capacity package" to realize the situation element display.
In large-scale three-dimensional scene display, application data model not only provides basic application data package, but also specifies the following knowledge implicitly: (1) basic structure of situation element orientated to application domain; (2) which segment is fixed in the situation element? Which segment is variable? What's the rule that variable segments abide by; (3) in human-computer interaction, the way to control the situation element, if the situation element need partial salient display or control classifiably, and the relation between display method and context scene.

Three-dimensional display software designers construct special "three-dimensional figure" according to these knowledge mentioned above. These "three-dimensional figure" must sufficiently use application situation element structure, especially the rule that variable elements abide by. Combining with 3D graphics drawing capacity package, designers construct real entity based on vertex data flow, line and vertex index set of plane figure and figure type.

This paper names the extended element display figure as "complex shape figure" for short, which is different from the basic figure such as point, line, triangle/plane, and triangle strip that basic $3 \mathrm{D}$ engine defined.

\section{Complex Shape Figure Class Design}

Some three-dimensional scene situation element display is simple. For example, locomotive target moving track of single line width can be directly realized by using the line, plane figure drawing interface provided by basic 3D engine. Some are complicated relatively. For example, radar power bubble of electromagnetic situation, information support chain related with weapon platform in joint operations, weapon action space based on different disturbance, etc. It's difficult to either express the complex three-dimensional display model by using the model file based on fixed triangle plane slice combined with DOF control, or realize it by directly using basic 3D engine drawing capacity package. It must transfer the vision effect to variable line, triangle plane slice set according to the structure of specific element. Then, the expectable situation element could be drawn by basic 3D engine.

In Fig 2, complex three-dimensional display model uses "complex figure drawing base class" (WS3dFigure) and specific figure drawing sub-class for different application requirement.

Base class provides virtual figure shape parameter setup interface and figure drawing interface. It can deploy vertex set, line/plane vertex index set data buffer of drawing figure aimed in specific 3D drawing engine. It supports figure data reusability in scene frame.

Sub-classes not only realize the interface of base class, but also save "figure shape parameter" to realize constructing the complex figure based on the data buffer of figure vertex set, line/plane vertex index set.

Fig. 2 shows the WS3dFigLinePAList class which supports the construction and display of locomotive target flight track, WS3dFigBProfBeam class which supports the display of radar detection beam and WS3dFigMProfCmxCone class which supports the display of radar dynamic detection sphere. 


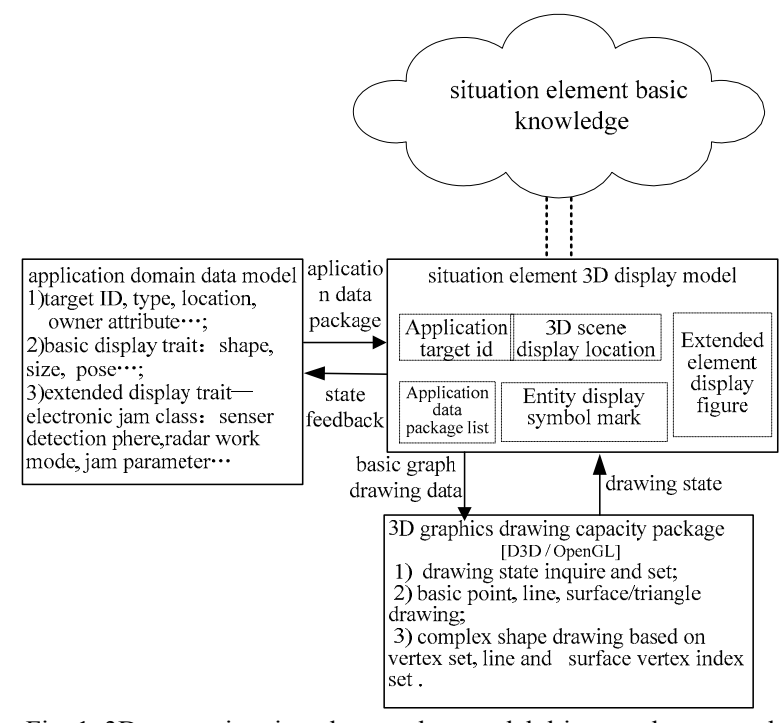

Fig. 1. 3D scene situation element data model driven and supported knowledge relation figure

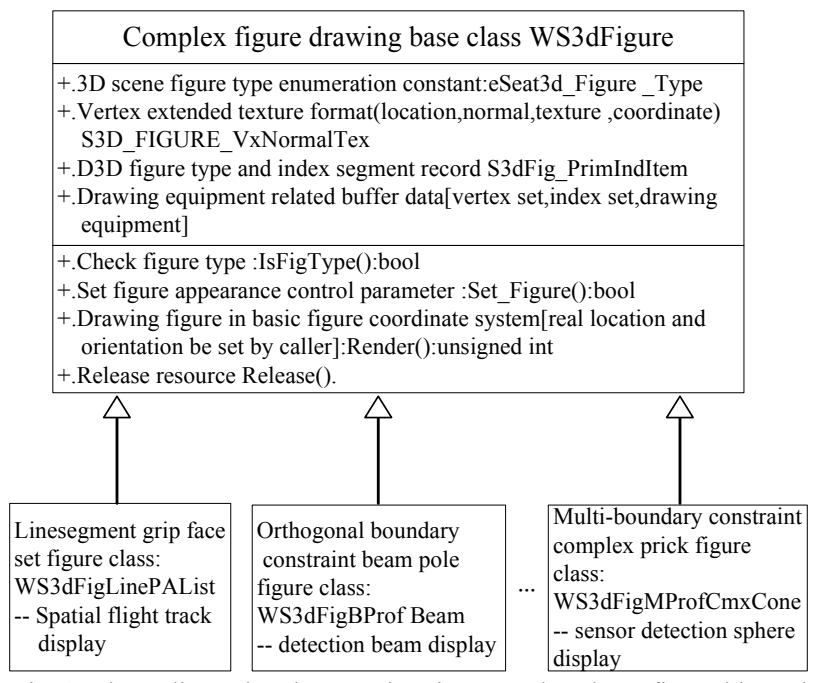

Fig. 2. Three-dimensional scene situation complex shape figure hierarchy



Fig. 3. Construction and drawing flow of complex shape

\section{E. Construction and Drawing of Complex Shape Figure Class}

In Fig. 3, complex shape figure drawing involves "check and construction of 3D display model" and "drawing complex figure in figure coordinate system". The "check and construction of 3D display model", combines with application situation element shape model knowledge, builds specific line, plane/triangle vertex set and vertex index set and saves these data set in buffer of $3 \mathrm{D}$ engine. In order to fully use the model which has been constructed in the previous frame, we can directly use the data set that is saved in buffer when the shape parameter, drawing color and texture are not changed. The "drawing complex figure in figure coordinate system" is simple. We can firstly set the state of drawing equipment. Then, we realize it by calling the figure drawing interface of basic $3 \mathrm{D}$ engine. The interface is based on data flow.

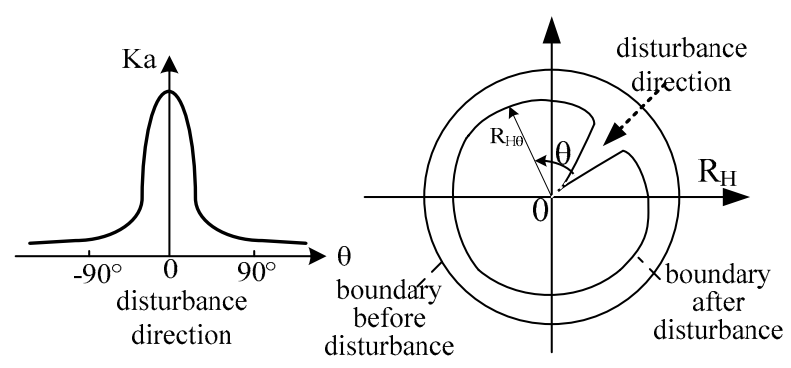

(a) direction coefficient on different disturbance degree

(b) E plane contrastive figure before and after disturbance

Fig. 4. Airborne radar E plane power sphere on disturbance

\section{CONSTRUCTION EXAMPLE OF THREE DiMENSIONAL DisPlay MODEL - RADAR POWER BUBble COMPLEX Figure DESIGN ON ELECTRONIC JAM}

In three-dimensional battlefield environment, electromagnetic situation element involves the visual description of radar spatial power sphere, shrinkage and gap display of power bubble on different disturbance mode and intensity. In the following text, firstly, combined with the display analysis of radar power bubble on electronic jam, the key data structure of radar power bubble three-dimensional display model is put forward,. Then, complex figure class is built. Finally, the drawing flow of situation element extended figure is introduced.

\section{A. Analyses of Application Domain Data Model and Description of Radar Power Bubble Structure}

Spatial power bubble of airborne radar is described by half-power point detection far boundary on $\mathrm{H}$ plane and $\mathrm{E}$ plane. $\mathrm{H}$ plane shows the detection far boundary of radar arbitrary azimuth vertical section. E plane shows the detection far boundary of certain height horizontal section. Electronic jammer usually adopts noise suppressible disturbance to airborne radar. In Fig. 4, radar E plane power sphere will change along with interference location and radar work parameter change, as well as notch location, notch width, and far boundary scale in all direction changing dynamically [8], [9].

Radar power bubble is reflected by two boundaries of $\mathrm{E}$ plane and $\mathrm{H}$ plane. In dynamic disturbance mode, he E plane boundary of radar power bubble is modulated gain on disturbance characteristic.

Application domain control model mainly involves the following three aspects:1) two boundaries data of radar power bubble E plane and $\mathrm{H}$ plane of system binding; 2) real-time varying radar spatial location and interference location; 3) dynamic varying interference disturbance 
intensity, disturbance frequency, disturbance mode and radar work mode.

Two boundaries of radar power bubble $\mathrm{E}$ plane, $\mathrm{H}$ plane and radar power bubble E plane boundary disturbance gain modulation can both be described by "spatial boundary profile segment sampling points set". See Fig. 5, the S3d_App_SpaceEdgeProf ("spatial boundary profile segment sampling points set") adopts 721 sampling points at most. It describes the far boundary distance based on radial angle on the elevation angle vertical section (or azimuth angle horizontal section). The interval between azimuth angles is $0.5^{\circ}$.If the last sampling point of boundary profile is the same as the beginning point, it denotes that the boundary profile is closed.

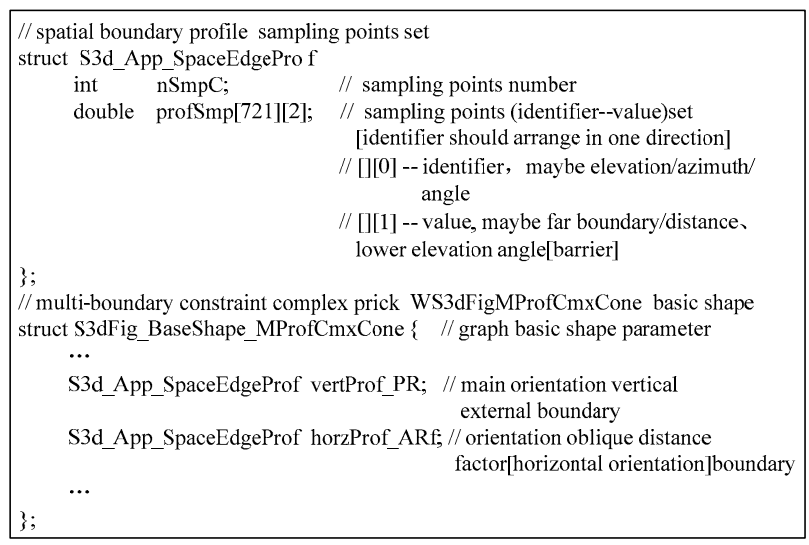

Fig. 5. Radar spatial power bubble data structure

\section{B. Building and Drawing Radar Power Bubble Three- Dimensional Model}

Radar power bubble can be drawn by plane-drawing. It also can be drawn by body-drawing. Plane-drawing transfers body data to an approximate plane. Therefore, we can make use of triangle or triangle trip drawing interface and the drawing capacity of basic three-dimensional engine. Body-drawing half-transparently projects the whole data scene to the 2D screen. It is independent of middle geometrical graphics [10], [11].This paper doesn't concern on body- drawing.

In Fig. 2, three-dimensional scene situation element display model adopts two hierarchies framework. That is "base class" and specific element figure "sub-class". Radar power bubble is expressed by "multi-boundary restricted complex prick envelope surface". The origin of complex prick is the same as the spatial location of airborne radar. The external envelope surface is a set of triangle strip. The triangle vertex is determined by azimuth angles, elevation angles and distance related to the original point of complex prick. The distance between vertices can be calculated by multiplying the vertical elevation varying factor, horizontal azimuth varying factor and the farthest distance. When the jammer is working, we firstly judge if the disturbance characteristic has been changed. If it has been changed, we correct the horizontal azimuth angle varying factor according to the disturbance characteristic after change.

We realize displaying the radar power bubble in scene drawing frame through drawing the "multi-boundary restricted complex prick envelope surface" in radar local coordinate system. The "multi-boundary restricted complex prick envelope surface" is just the three-dimensional display model of radar power bubble.

The main steps of drawing the "multi-boundary restricted complex prick" are 1) when sensor (radar) is original created, setup the oblique distance of boundary sampling points on $\mathrm{H}$ plane and E plane 2) before drawing in every frame, check and correct the azimuth oblique distance correcting factor based on basic disturbance parameter 3) modify the equipment drawing matrix and transfer the world coordinate system to the sensor local coordinate system 4) draw the radar power bubble, check the shape parameter, trigger constructing drawing geometry, and then use D3D to draw the complex figure based on vertex set and plane vertex index set 5) restore the equipment drawing matrix and state.

\section{Multi-view display effect of airborne radar power bubble on dynamic electronic jam}

In a certain idea demo, we firstly draw the jammer, the platform of airborne radar and the "complex prick envelope surface" of radar power bubble. Then, according to the jammer location and disturbance intensity, we construct and update the "complex prick envelope surface" of radar power bubble. In this way, we realize the three-dimensional expression of airborne radar dynamic detection sphere on electronic jam. Fig 6 shows an airborne radar power bubble before and after dynamic disturbance. Fig 7 shows the power surface triangle subdivision of airborne radar after dynamic disturbance.

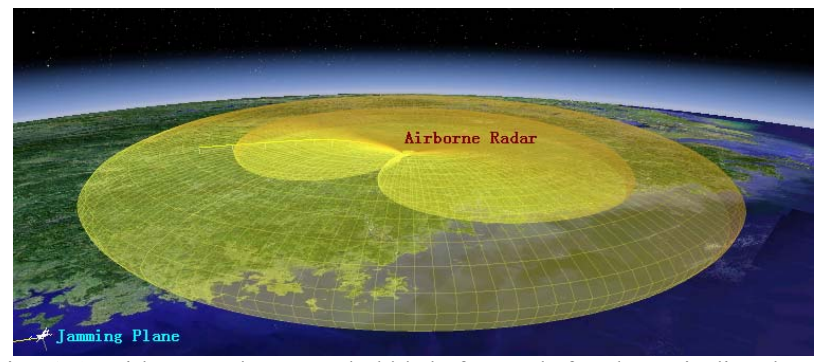

Fig. 6. An airborne radar power bubble before and after dynamic disturbance

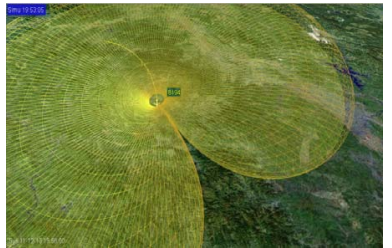

(a) observe from notch

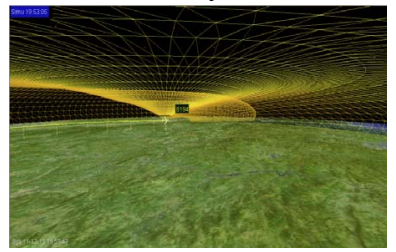

(b) observe from power bubble inner
Fig. 7. the detection sphere surface triangle subdivision after disturbance

\section{CONCLUSION}

The visual expression of situation element is important in three-dimensional battlefield environment. Entity object can be expressed by adopting fixed shape two-dimensional, three-dimensional symbol or three-dimensional geometrical model saved in model file. However, many situation elements must modify the presentation character such as the display shape, color and lightness according to dynamic changing foe or friend situation. The dynamic complex situation element display the idea that "three-dimensional display model construction" not only can inherit the method of displaying entity symbol or three-dimensional geometrical model based on model file, but also can draw complex three-dimensional geometrical figure in time based on 
dynamic varying situation data. It elementarily realizes displaying and updating complex situation element of large scale virtual scene.

Three-dimensional battlefield environment situation element isn't independent. The situation element display model must automatically modify display presentation, display granularity based on environment context. We just take a try. It needs continuative study on how to make the situation element display more visualize, how to improve the drawing speed, how to realize intelligent choice and interaction based on three-dimensional scene situation element, etc.

\section{REFERENCES}

[1] X. J. Liao, R. F. Wang, Digital battlefield Visualization Technique and Application, National defense industry publishing company, pp. 5-6, pp. 17-18, pp. 272-277, 2010.

[2] D. Shreiner, M. Woo, J. Neider, T. Davis, and X. B, OpenGL program guide(the fifth edition), Machine industry publishing company, Jun 2006. pp. 1-3. pp. 23-60.

[3] D. C. Wang, G. S. Yang, and Y. P. Sun, Proficiency of Direct3D graphics and animation program design, BeiJing, dumb millions post and electronic publishing company, May 2007. pp. 3-5, pp. 49-60.

[4] X Files (Legacy). (Apr 2005). 3D Studio File Format Information. DirectX9.chm. [Online]. Available: http://www.mediatel.lu

[5] Advanced Visualization Option Help, V0.chm, STK V6.0 Online help. Oct, 2004.

[6] Multigen Paradigm Cop, Creator_Help.chm, Nov 2004.
[7] D. Li and X. G. Liu, "Modern radar anti- jamming efficiency based on simulation," Command Information system and technology, vol. 1, pp. 70-75, 2011.

[8] S. J. Mao, Z. Q. Ju, and Y. P. Li, C4ISR System Simulation Experiment Technique, Military Science publishing company, 2011, ch. 1, pp. 118.

[9] Y. Z. Wang, X. H. Zhang, and X. J. Zhang, "Study on battlefield electromagnetic environment visualize," Modern recovery technology, vol. 8, pp. 38-42, Dec 2004.

[10] Q. Zhou, J. W. Chen, and J. S. Li, "Electromagnetic environment building and 3D visualize," Mapping science and technology transaction, vol. 6, pp. 112-115, Apr 2008.

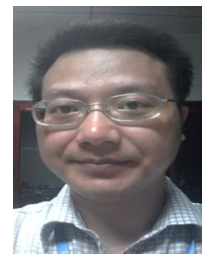

Chen Rong was born in Yancheng city, Jiang Su province, the people's republic of China. He graduated at Zhejiang University and got the computer science \& technology doctor degree at 2005. And he has worked for CETC 28th Institution, and engages in complex system integration job.

Wang Xian Jin received the B.Eng. and M.Eng. degree from the Missile College of AirForce Engineering University, Xi' an, China in 1989 and 1995, respectively, all in computer application. From 2005 to now, he works as software engineer in the CETC 28th Research institution.

Chen Jie received the Computer Science \&Technology Master degree from Nanjing University of Aeronautics and Astronautics, NanJing,China,in 2011. And she works as software engineer in the CETC 28th Research institution. 